\title{
SECADO DE FORRAJE CON EL HORNO MICROONDAS: EFECTO SOBRE EL ANÁLISIS DE CALIDAD
}

\author{
Microwave oven forage drying: effects on quality analysis
}

\author{
Roberto J. Crespo $^{1}$ *, Jorge A. Castaño ${ }^{2}$ y José A. Capurro ${ }^{3}$
}

\begin{abstract}
A B S T R A C T
The objectives were to utilize a microwave oven (HM) to determine dry matter (MS) and evaluate its effect on dry matter (MS), organic matter content $(\mathrm{MO})$, in vitro organic matter digestibility (DIVMO), brute protein (PB), acid detergent fiber (FDA), and acid-detergent insoluble nitrogen (NIDA). We utilized the forage species: Medicago sativa L., Trifolium repens L., Trifolium pratense L. and Thinopyrum ponticum Barkw. \& D.R. Dewey, and mixtures of: M. sativa-Dactylis glomerata L., Festuca arundinacea Schreb.-T. repens-D. glomerata, and Lolium perenne L.-T. repens. The experimental design was a factorial complete randomized block. We compared drying time (Ts) and quality parameters on stove method (T1), HM at $900 \mathrm{~W}$ (T2), and $\mathrm{HM}$ at 900 and $400 \mathrm{~W}$ (T3), by ANDEVA and Duncan Test ( $\mathrm{p} \leq 0.05)$. In T2 and T3, Ts ranged from 6 to 8 min (T. pratense and Th. ponticum, respectively), compared to $48 \mathrm{~h}$ for $\mathrm{T} 1$. The MS value did not show differences between treatments for $M$. sativa, T. pratense, M. sativa-D. glomerata, and $L$. perenne-T. repens, but there were differences for the other forages between $\mathrm{T} 2$ or $\mathrm{T} 3$ with $\mathrm{T} 1$, which could be explained by the forages phenological stage. Differences between treatments were found for FDA (T. repens), DIVMO and PB (Th. ponticum), and PB and MO ( $L$. perenne-T. repens). The use of the microwave oven allows a quick and consistent MS determination, along with a reliable forage quality standard evaluation.
\end{abstract}

Key words: dry matter, stove, drying time, brute protein, acid detergent fiber.

\section{R E S U M E N}

Los objetivos de este experimento fueron utilizar el horno microondas (HM) para determinar materia seca (MS) y evaluar su efecto sobre el valor de materia orgánica (MO), digestibilidad in vitro de la MO (DIVMO), proteína bruta (PB), fibra detergente ácido (FDA) y nitrógeno insoluble en detergente ácido (NIDA). Se utilizaron forrajes puros: Medicago sativa L., Trifolium repens L., Trifolium pratense L. y Thinopyrum ponticum Barkw. \& Dewey, y en mezclas: M. sativa-Dactylis glomerata L., Festuca arundinacea Schreb.- T. repens-D. glomerata, y Lolium perenne L.-T. repens. El diseño experimental usado fue bloques completamente aleatorizados con arreglo factorial de tratamientos. Se comparó el tiempo de secado (Ts) y los parámetros de calidad entre: Estufa (T1), HM a 900 W (T2), y HM a 900 y 400 W (T3), mediante ANDEVA y Test de Duncan ( $\mathrm{p} \leq 0,05)$. Para T2 y T3, Ts varió entre 6 a $8 \mathrm{~min}$ (T. pratensey Th. ponticum respectivamente), siendo en T1, $48 \mathrm{~h}$. La MS no difirió entre tratamientos para $M$. sativa, T. pratense, M. sativa-D. glomerata y $L$. perenne-T. repens, pero si en el resto de los forrajes entre T2 ó T3 con T1; esto se podría asociar al estado fenológico del material forrajero al momento del corte. Respecto a los parámetros de calidad evaluados se observaron diferencias en FDA en T. repens, DIVMO y PB en Th. ponticum, y PB y MO en $L$. perenne-T. repens. El uso del HM permite obtener rápida y confiablemente la MS del forraje sin modificar sustancialmente sus parámetros de calidad.

Palabras clave: materia seca, estufa, tiempo de secado, proteína bruta, fibra detergente ácida.

\footnotetext{
${ }^{1}$ Universidad Nacional del Centro de la Provincia de Buenos Aires, Facultad de Agronomía, Av. República de Italia 780, C.C.47. CP 7300. Azul, Buenos Aires, Argentina. E-mail: rojacre@yahoo.com.ar *Autor para correspondencia.

${ }^{2}$ Instituto Nacional de Tecnología Agropecuaria, Estación Experimental Agropecuaria Balcarce, Ruta Nacional 226, km 73,5, C.C. 276. CP 7620, Balcarce, Buenos Aires, Argentina. E-mail: jcastanio@balcarce.inta.gov.ar

${ }^{3}$ Universidad Nacional de Mar del Plata, Facultad de Ciencias Agrarias, Ruta Nacional 226, km 73,5, C.C. 276.

CP 7620, Balcarce, Buenos Aires, Argentina. E-mail: jcapurro@balcarce.inta.gov.ar

Recibido: 1 de febrero de 2006. Aprobado: 29 de mayo de 2006.
} 


\section{INTRODUCCIÓN}

El valor de materia seca (MS) ha sido utilizado hasta la actualidad como el parámetro con el que se expresan resultados de producción y evaluación del recurso forrajero (Crespo, 2002). Dicho valor se encuentra afectado por el valor de materia verde, el cual depende del contenido de humedad del forraje y varía con la especie, el estado fenológico y en menor medida con la estación del año (Bruno et al., 1995; Agnusdei et al., 2001).

En varios trabajos de investigación se ha demostrado que la utilización de la estufa de circulación forzada de aire es satisfactoria como método para obtener el valor de MS (Narasimhalu et al., 1982; Higgins y Spooner, 1986). Por su lentitud en el proceso de secado, da lugar a la continuidad del proceso de respiración enzimática en los tejidos vegetales y posible volatilización de ácidos orgánicos y amonio (Narasimhalu et al., 1982) con la consecuente alteración de su composición química (Higgins y Spooner, 1986; Hernández Pastorini et al., 2002). Por otro lado, para el secado convencional de los forrajes, la estufa requerida no es de presencia común en los establecimientos agropecuarios (Benvenutti y Cavalli, 1994; Petruzzi et al., 2005).

Con la finalidad de mejorar la rapidez en la determinación del valor de MS se recurre a otros métodos alternativos a la estufa, entre los que se incluye al horno microondas (Benvenutti y Cavalli, 1994; Crespo, 2002). Sin embargo, existe limitada información sobre el secado de forrajes con el horno microondas y su posible efecto sobre la calidad del mismo (Narasimhalu et al., 1982; Crespo, 2002).

En el presente trabajo se propone como hipótesis que la utilización del método de secado con el horno microondas permitiría obtener valores de MS similares a los obtenidos con la estufa de circulación de aire forzada, con una mayor rapidez y sin alterar los parámetros de calidad del forraje.

Los objetivos que se plantearon fueron: a) calibrar el método de secado con horno microondas para distintos materiales forrajeros; b) comparar el método de secado con estufa de circulación forzada de aire versus horno microondas; y c) determinar la posible alteración con el horno microondas, de ciertos parámetros estándar de la calidad en distintos materiales forrajeras.

\section{MATERIALES Y MÉTODOS}

Se utilizaron siete materiales forrajeros: cuatro compuestos por especies forrajeras puras y tres por pasturas polifíticas, extraídas y analizadas en la Unidad Integrada Balcarce, de la Estación Experimental Agropecuaria Balcarce del Instituto Nacional de Tecnología Agropecuaria (INTA) - Facultad de Ciencias Agrarias de la Universidad Nacional de Mar del Plata, durante el año 2003. Los materiales fueron: alfalfa pura (Medicago sativa $\mathrm{L}$.); trébol blanco puro (Trifolium repens L.); trébol rojo puro (Trifolium pratense L.); agropiro alargado puro [Thinopyrum ponticum (Podp.) Barkw. \& D.R. Dewey]; mezcla en partes iguales de alfalfa y pasto ovillo (Dactylis glomerata L.); mezcla de festuca (Festuca arundinacea Schreb.), trébol blanco y pasto ovillo en una proporción del 60,35 y $5 \%$ respectivamente, y mezcla de ryegrass perenne ( $\mathrm{Lo}$ lium perenne L.) y trébol blanco, en proporción 70 y $30 \%$, respectivamente.

Todos los materiales se cortaron a ras del suelo con una tijera manual tipo "esquila", y sin separar el material vivo del muerto se trozaron para ser secados. Esto determinó que las muestras de T. repens, $T$. pratense, L. perenne y Th. ponticum, que se encontraban en estadíos vegetativos y de floración (Bridke, 1991), hayan sido más heterogéneas. Por otro lado, el resto de los materiales forrajeros estaban solamente en estado vegetativo (Bridke, 1991), lo que determinó una muestra más homogénea, sólo con material vegetal vivo. El trozado previo de 4 a $5 \mathrm{~cm}$ de tamaño se realizó con el propósito de que la muestra de material forrajero entrara en la bandeja plástica utilizada para secar las muestras. Estas bandejas son de polipropileno, especiales para el uso con alimentos en horno de microondas, con un volumen aproximado de $400 \mathrm{~cm}^{3}$.

Se definieron tres tratamientos: T1: secado en estufa a $65^{\circ} \mathrm{C}$ con circulación forzada de aire o control; T2: secado en horno microondas a una potencia de $900 \mathrm{~W}$; y T3: secado en horno microondas alternando 900 y $400 \mathrm{~W}$ de potencia en el secado de la misma muestra. A la finalización de cada tratamiento, se pesó la muestra de forraje seca y se estimó el \% MS por relación con el peso previo al secado.

Se tomó T1 como tratamiento control, conducido según el procedimiento de la norma S358.2 de 
ASAE (2004). Se utilizó una estufa con circulación de aire forzado (Unitherm Drying Oven, de S\&T Engineering Company, Birmingham, Inglaterra), de 0 a $120^{\circ} \mathrm{C}$ de temperatura y $220 \mathrm{~V}$ a 50 ciclos. La muestra de forraje se colocó directamente en bandejas metálicas con un volumen de $4.500 \mathrm{~cm}^{3}$, construidas en aluminio con una malla del mismo material en su fondo para permitir la circulación de aire.

Los procedimientos seguidos para los tratamientos T2 y T3 fueron llevados a cabo según la metodología informada por Crespo (2002). Según éste, la muestra de forraje debe ser sometida a un tiempo y a una potencia programados en el horno, y observar cuál es el efecto de la radiación emitida sobre la muestra. Cada muestra de forraje fue introducida en el microondas sobre una bandeja previamente tarada, y sometida a distintos ciclos de secado. La duración del ciclo de secado fue la correcta cuando, al mayor tiempo posible determinado en el contador electrónico del horno microondas y a una potencia predeterminada, la muestra no se carbonizó.

Se consideró que la muestra alcanzó su peso seco final, cuando llegó a peso constante luego de tres pesadas consecutivas. A partir de aquí el tiempo total de secado se obtuvo de la suma de los ciclos de secado, considerándose como última a la primer tanda de secado en que se registró el valor de peso constante (Crespo, 2002).

En T3 se buscó evaluar el efecto del cambio de potencia y tiempo de tanda de secado sobre las distintas especies forrajeras utilizadas. Para ello, en la primera etapa se trabajó a potencia máxima $(900$ W) por un ciclo de tiempo de tiempo máximo y que dependió de la especie forrajera a evaluar; en la segunda etapa se trabajó a mediana potencia ( 400 W) y los tiempos se definieron por observación directa (Crespo, 2002).

Dentro del horno microondas se colocó un vaso con $150 \mathrm{~cm}^{3}$ de agua aproximadamente, de manera de humedecer el medio y evitar la ignición de la muestra cuando se avanza en el proceso de secado. Cuando se finalizó cada ciclo de secado, se removió la muestra de forraje con el fin de lograr uniformidad de secado. Asimismo, se descartó el agua del vaso y se reemplazó por igual cantidad, pero a temperatura ambiente, para evitar la ebullición y proyección sobre la muestra.
Se utilizó un horno microondas (Moulinex Óptimo, Buenos Aires, Argentina) con una potencia máxima de $900 \mathrm{~W}$ y mínima de $75 \mathrm{~W}$, a una frecuencia de 2.450 MHz y cronómetro digital prefijable descendente, que facilitó la toma de los tiempos. Se realizaron 10 repeticiones por tratamiento, sumando 30 muestras por material forrajero, siendo el peso de cada una de ellas de $50 \mathrm{~g}$ determinado por el volumen de la bandeja plástica. Se utilizó una balanza digital (COBOS M-200, Barcelona, España) con máximo de pesada de $200 \mathrm{~g}$ y una precisión de $0,01 \mathrm{~g}$.

En las muestras secas se determinó materia orgánica (MO), digestibilidad in vitro de la materia orgánica (DIVMO), proteína bruta (PB), fibra detergente ácido (FDA) y nitrógeno insoluble en detergente ácido (NIDA).

La MO se determinó por diferencia entre pesajes de la muestra antes y después de la calcinación en horno mufla a $600^{\circ} \mathrm{C}$ durante $6 \mathrm{~h}$ (Tilley y Terry, 1963), mientras que la DIVMO se calculó por diferencia de pesajes antes y después de la calcinación de los residuos de la digestibilidad in vitro de la MS por Tilley y Terry (1963).

La PB se obtuvo por combustión en atmósfera con oxígeno ultra puro, posterior análisis de $\mathrm{N}$ por el método de Kjeldahl y multiplicación por el factor 6,25 (Horneck y Miller, 1998). Por otro lado, la FDA se determinó por el método de las bolsitas filtrantes (Komarek et al., 1994) y el NIDA determinando PB en la muestra y en el residuo de la misma tratada con detergente ácido, para lo cual se utilizaron las técnicas anteriores de PB y FDA (Komarek et al., 1994; Horneck y Miller, 1998).

Los resultados obtenidos se analizaron por análisis de varianza (ANDEVA), para evaluar el efecto del tratamiento sobre las variables respuestas porcentaje de MS y tiempo de secado obtenidos, bajo un diseño completamente aleatorizado con arreglo factorial de tratamientos. Las medias se contrastaron por medio del Test de Duncan ( $\mathrm{p} \leq 0,05)$.

\section{RESULTADOS Y DISCUSIÓN}

\section{Determinación del tiempo de cada fase del ciclo de secado con el método de horno microondas Crespo (2002) encontró diferencias significativas entre los tiempos de secado de los distintos materia- les con que trabajó, por lo que se hizo necesario ade- cuar los tiempos a los materiales aquí utilizados.}


En el Cuadro 1 se observan los tiempos de cada fase del ciclo completo de secado necesarios para obtener los porcentajes de MS de los materiales forrajeros utilizados. Se considera una fase del ciclo de secado al tiempo máximo que una muestra de forraje resiste la acción de las microondas sin que se carbonice. La carbonización se manifestó cuando la muestra viró de color verde a gris (apreciación visual), y/o se detectó aroma a quemado (apreciación olfativa).

En T2 se comenzó con tiempos de fase de secado de 6 min, luego de $5 \mathrm{~min}$, y se observó que en cualquiera de ellas los materiales forrajeros se carbonizaban. Salvo Th. ponticum, que cuando se expuso durante 5 min no evidenció problema alguno de carbonización, el resto de los materiales evaluados toleraron un máximo de 4 min como tiempo inicial de fase de secado.

Para el mismo tratamiento, la segunda fase de secado fue de $1 \mathrm{~min}$ en todos los materiales. En Th. ponticum se repitieron fases de $1 \mathrm{~min}$ de duración, hasta peso constante. Asimismo, en M. sativa las fases de secado sucesivas fueron de $30 \mathrm{~s}$ hasta la misma condición.

En T. repens, T. pratense y las tres mezclas forrajeras se realizó una tercera fase de secado de $1 \mathrm{~min}$ de duración, para luego culminar el proceso con fases de secado sucesivas de $30 \mathrm{~s}$ hasta peso constante.

En T3, cuando se programó el horno a $900 \mathrm{~W}$ se comenzó con fases de secado de igual duración que en T2. Luego se programó a $400 \mathrm{~W}$ de potencia y se ejecutaron dos fases de secado consecutivas de 1 min de duración cada una en M. sativa, y una de 2 min más otra de 1 min en el resto de los materiales forrajeros. Por último, en T3 se realizaron fases de secado de $30 \mathrm{~s}$ a la menor potencia para todos los materiales utilizados hasta peso constante.

\section{Determinación del contenido de MS en distintos materiales forrajeros}

Con el objetivo de facilitar el análisis de los distintos materiales forrajeros utilizados, los resultados se presentan por separado, agrupando por un lado los materiales forrajeros puros y por el otro, los compuestos por pasturas polifíticas.

a) Evaluación del secado con horno microondas utilizando especies puras. El horno microondas redujo significativamente el tiempo de secado en todos los materiales forrajeros evaluados respecto al método de la estufa (Cuadro 2). Estos resultados coinciden con los obtenidos por Crespo (2002) y

\section{Cuadro 1. Tiempo de cada fase del ciclo de secado determinado con el horno microondas para los distintos materiales forrajeros.}

Table 1. Forage drying turn around times for the different materials determined using the microwave oven.

\begin{tabular}{lccccc}
\hline \multirow{2}{*}{ Material forrajero } & Trat. & \multicolumn{3}{c}{$\mathbf{N}^{\mathbf{0}}$ de fase del ciclo de secado (min) } \\
\cline { 2 - 6 } & & $\mathbf{1}^{\text {ra }}$ & $\mathbf{2}^{\text {da }}$ & $\mathbf{3}^{\text {ra }}$ & $\mathbf{n}^{\mathbf{1}}$ \\
\hline M. sativa & T2 & 4 & 1 & - & 0,5 \\
T. repens & T3 & 4 & 1 & 1 & 0,5 \\
\multirow{2}{*}{ T. pratense } & T2 & 4 & 1 & 1 & 0,5 \\
Th. ponticum & T3 & 4 & 2 & 1 & 0,5 \\
M. sativa-Dactylis glomerata & T2 & 4 & 1 & 1 & 0,5 \\
F. arun.-T. repens-D. glom. & T3 & 4 & 2 & 1 & 0,5 \\
\multirow{2}{*}{ L. perenne-T. repens } & T2 & 5 & - & - & 1 \\
& T3 & 5 & 2 & 1 & 0,5 \\
& T2 & 4 & 1 & 1 & 0,5 \\
& T3 & 4 & 2 & 1 & 0,5 \\
& T2 & 4 & 1 & 1 & 0,5 \\
& T3 & 4 & 2 & 1 & 0,5 \\
& T2 & 4 & 1 & 1 & 0,5 \\
& T3 & 4 & 2 & 1 & 0,5 \\
\hline
\end{tabular}

\footnotetext{
${ }^{1}$ Representa el tiempo, en minutos, de cada fase de ciclo de secado, posterior a las tres primeras hasta llegar a peso constante. D. glom.: Dactylis glomerata; F. arun.: F. arundinacea.

T2: tratamiento 2; T3: tratamiento 3.
} 
Cuadro 2. Tiempos de secado (h) de los tratamientos utilizando especies forrajeras puras.

Table 2. Drying time (h) by treatment using single species.

\begin{tabular}{lrrrr}
\hline \multicolumn{1}{c}{ Tratamiento } & \multicolumn{4}{c}{ Especie pura } \\
\cline { 2 - 5 } & \multicolumn{1}{c}{ M.s. } & \multicolumn{1}{c}{ T.r. } & T.p. & \multicolumn{1}{c}{ Th.p. } \\
\hline T1 Estufa & $48 \mathrm{a}$ & $48 \mathrm{a}$ & $48 \mathrm{a}$ & $48 \mathrm{a}$ \\
T2 Microondas $900 \mathrm{~W}$ & $0,107 \mathrm{~b}$ & $0,115 \mathrm{c}$ & $0,107 \mathrm{c}$ & $0,097 \mathrm{c}$ \\
T3 Microondas $900-400 \mathrm{~W}$ & $0,110 \mathrm{~b}$ & $0,125 \mathrm{~b}$ & $0,132 \mathrm{~b}$ & $0,122 \mathrm{~b}$ \\
\hline
\end{tabular}

Los valores en columnas seguidos de una misma letra no difieren entre sí según Test de Duncan ( $\mathrm{p} \leq 0,05)$.

M.s.: Medicago sativa, T.r.: Trifolium repens; T.p.: Trifolium pratense, Th.p.: Thinopyrum ponticum.

otros autores como Farmer y Brusewitz (1980), Schuman y Rauzi (1981), Benvenutti y Cavalli (1994), Hernández Pastorini et al. (2002) y Petruzzi et al. (2005).

En el Cuadro 2 se observa que en $M$. sativa no existieron diferencias significativas $(p>0,05)$ entre $\mathrm{T} 2$ y T3, pero sí difirieron $(\mathrm{p} \leq 0,05)$ de $\mathrm{T} 1$. En el resto de las especies evaluadas los tratamientos difirieron $(\mathrm{p} \leq 0,05)$ entre sí en los tiempos de secado obtenidos. La mayor homogeneidad de la muestra en relación al balance entre material vegetal vivo y muerto, podría explicar la diferencia entre los resultados obtenidos en $M$. sativa respecto a los otros materiales puros utilizados (Cuadro 3).

En el mismo cuadro (Cuadro 2) se aprecia que en todos los materiales el tratamiento T2 insumió un menor tiempo respecto a $\mathrm{T} 3$ para secar la muestra de forraje (entre 3 y $20 \%$ menor en M. sativa y Th. ponticum, respectivamente), variando entre $7 \mathrm{~min}$ como máximo y 6 min como mínimo en $T$. repens y $T h$. ponticum, respectivamente. Estos resultados están dentro de lo esperable, ya que la utilización de una mayor potencia en el horno microondas, reduce el tiempo de secado del forraje (Farmer y Brusewitz, 1980; Benvenutti y Cavalli, 1994; Crespo 2002).
En el Cuadro 3 se presentan los valores de porcentaje de MS obtenidos con los distintos tratamientos en las especies puras utilizadas y sus respectivos coeficientes de variación (porcentaje de $\mathrm{CV}$ ). Se observa que en $M$. sativa y $T$. pratense no existen diferencias significativas $(\mathrm{p} \leq 0,05)$ entre los porcentajes de MS obtenidos con cualquiera de los tratamientos. Para alfalfa, Petruzzi et al. (2005) utilizaron un horno microondas de similar potencia $(850 \mathrm{~W})$ e igual tamaño de muestra de forraje, y no obtuvieron una buena correlación entre el porcentaje de MS obtenido con el horno microondas y la estufa.

En T. repens no existieron diferencias significativas $(p>0,05)$ entre T1 y T2, pero sí difirieron de T3 (Cuadro 3). Estas diferencias se podrían deber a la alta heterogeneidad del material en cuanto a contenido de material vivo y muerto. Esta suposición está parcialmente avalada por el mayor coeficiente de variación (CV) que se aprecia entre las muestras de este material. Asimismo, con T. ponticum, T1 no difirió de T3 pero sí de T2 (Cuadro 3).

b) Evaluación del secado con horno microondas utilizando pasturas polifíticas. En el Cuadro 4 se visualizan los tiempos necesarios para secar las

Cuadro 3. Materia seca (\%) y coeficientes de variación (CV) de forrajeras puras bajo distintos tratamientos. Table 3. Dry matter (\%) and variation coefficient $(\mathrm{CV})$ of single species by treatment.

\begin{tabular}{lcccc}
\hline \multirow{2}{*}{ Tratamiento } & \multicolumn{4}{c}{ Especie pura } \\
\cline { 2 - 5 } & M.s. & T.r. & T.p. & Th.p. \\
\hline T1 Estufa & $16,64 \mathrm{a}$ & $22,61 \mathrm{~b}$ & $20,56 \mathrm{a}$ & $27,18 \mathrm{a}$ \\
T2 Microondas 900 W & $17,33 \mathrm{a}$ & $22,97 \mathrm{~b}$ & $21,29 \mathrm{a}$ & $26,59 \mathrm{~b}$ \\
T3 Microondas 900 - 400 W & $17,05 \mathrm{a}$ & $26,43 \mathrm{a}$ & $20,39 \mathrm{a}$ & $26,76 \mathrm{ab}$ \\
CV $(\%)$ & 2,04 & 8,79 & 2,3 & 1,13 \\
\hline
\end{tabular}

Los valores en columnas seguidos de una misma letra no difieren entre sí según Test de Duncan ( $\mathrm{p} \leq 0,05)$.

M.s.: Medicago sativa; T.r.: Trifolium repens; T.p.: Trifolium pratense; Th.p.: Thinopyrum ponticum. 
Cuadro 4. Tiempos de secado (h) de distintos tratamientos utilizando forrajes en mezcla.

Table 4. Forage mixture drying time (h) by treatment.

\begin{tabular}{lrrr}
\hline \multirow{2}{*}{ Tratamiento } & \multicolumn{3}{c}{ Mezcla forrajera } \\
\cline { 2 - 4 } & M.s.-D.glom. & F.arun.-T.r.-D.glom. & L.p.-T.r. \\
\hline T1 Estufa & $48 \mathrm{a}$ & $48 \mathrm{a}$ & $48 \mathrm{a}$ \\
T2 Microondas 900 W & $0,101 \mathrm{c}$ & $0,094 \mathrm{c}$ & $0,098 \mathrm{c}$ \\
T3 Microondas 900 - 400 W & $0,113 \mathrm{~b}$ & $0,120 \mathrm{~b}$ & $0,117 \mathrm{~b}$ \\
\hline
\end{tabular}

Los valores en columnas seguidos de una misma letra no difieren entre sí según Test de Duncan ( $\mathrm{p} \leq 0,05)$.

M.s.: Medicago sativa; D.glom.:Dactylis glomerata; F.arun.: Festuca arundinacea; T.r.: Trifolium repens; L.p.: Lolium perenne; T.r.: Trifolium repens.

pasturas polifíticas evaluadas. En el tratamiento con estufa (T1), el tiempo de secado fue considerablemente superior al necesario en el resto de los tratamientos.

También se observa que existieron diferencias significativas $(\mathrm{p} \leq 0,05)$ entre los tratamientos con horno de microondas. En los tres materiales forrajeros utilizados $\mathrm{T} 3$ registró un mayor tiempo de secado respecto a T2 $(11 ; 22$ y $17 \%$ mayor en $M$. sativa-D. glomerata, $F$. arundinacea-T. repens- $D$. glomerata y $L$. perenne-T. repens, respectivamente, según el orden del Cuadro 4. Se aprecian valores máximos de 7,5 min, mientras que el mínimo fue de poco más de 6 min (Cuadro 4).

Los tiempos de secado registrados con el horno microondas a distintas potencias exhiben igual tendencia que los obtenidos en los trabajos de Farmer y Brusewitz (1980), Benvenutti y Cavalli (1994) y Crespo (2002), ya que al utilizar una mayor potencia se reduce el tiempo de secado.

En el Cuadro 5 se observan los valores de MS obtenidos con los distintos tratamientos, utilizando materiales forrajeros en mezclas de especies. También se aprecian los bajos valores de CV obtenidos.
Los porcentajes de MS obtenidos en la mezcla $M$. sativa-D. glomerata y $L$. perenne-T. repens no evidenciaron diferencias significativas $(\mathrm{p} \leq 0,05)$ entre los distintos tratamientos. Por otro lado, en $F$. arundinacea-T. repens-D. glomerata el valor de porcentaje de MS obtenido con los tratamientos T1 y T2 fue similar $(\mathrm{p} \leq 0,05)$ pero difirió del obtenido con T3 (Cuadro 5).

\section{Determinación de parámetros de calidad en los materiales forrajeros}

En el Cuadro 6 se presentan los valores de algunos parámetros de calidad del forraje obtenidos para los distintos tratamientos, utilizando materiales forrajeros puros y en mezclas. Salvo casos aislados, no se registraron diferencias $(p>0,05)$ en el valor de los parámetros de calidad evaluados para los diferentes materiales forrajeros en función del método de secado empleado. En los casos donde se detectaron diferencias, éstas se atribuyen a la heterogeneidad del material en estudio ( $T$. repens florecido, Th. ponticum en elongación) (Cuadro 6).

En $M$. sativa los resultados obtenidos concuerdan con los de Higgins y Spooner (1986), quienes evaluaron el secado en estufa de circulación forzada de aire por $72 \mathrm{~h}$ a $50^{\circ} \mathrm{C}$, y en microondas por $7 ; 8$;

Cuadro 5. Materia seca (\%) y coeficientes de variación (CV) de mezclas forrajeras bajo distintos tratamientos. Table 5. Dry matter (\%) and variation coefficient (CV) of forage mixture by treatment.

\begin{tabular}{lccc}
\hline Tratamiento & \multicolumn{3}{c}{ Mezcla forrajera } \\
\cline { 2 - 4 } & M.s.-D.glom. & F.arun.-T.r.-D.glom. & L.p.-T.r. \\
\hline T1 Estufa & $17,12 \mathrm{a}$ & $24,96 \mathrm{a}$ & $22,86 \mathrm{a}$ \\
T2 Microondas 900 W & $16,96 \mathrm{a}$ & $24,98 \mathrm{a}$ & $23,09 \mathrm{a}$ \\
T3 Microondas $900-400 \mathrm{~W}$ & $17,45 \mathrm{a}$ & $23,43 \mathrm{~b}$ & $22,49 \mathrm{a}$ \\
CV $(\%)$ & 1,45 & 3,64 & 1,33 \\
\hline
\end{tabular}

Los valores en columnas seguidos de una misma letra no difieren entre sí según Test de Duncan ( $\leq 0,05)$.

M.s.: Medicago sativa; D.glom.: Dactylis glomerata; F.arun.: Festuca arundinacea; T.r.: Trifolium repens; L.p.: Lolium perenne; T.r.: Trifolium repens. 
Cuadro 6. Parámetros de calidad de distintos forrajes sometidos a distintos tratamientos de secado.

Table 6. Quality parameters of the forage under different drying treatments.

\begin{tabular}{|c|c|c|c|c|c|c|}
\hline $\begin{array}{l}\text { Material } \\
\text { forrajero }\end{array}$ & Tratamiento & $\begin{array}{l}\text { MO } \\
(\%) \\
\end{array}$ & $\begin{array}{c}\text { DIVMO } \\
(\%) \\
\end{array}$ & $\begin{array}{l}\text { PB } \\
(\%)\end{array}$ & $\begin{array}{c}\text { FDA } \\
(\%) \\
\end{array}$ & $\begin{array}{c}\text { NIDA } \\
(\%)\end{array}$ \\
\hline \multirow{3}{*}{ M.s. } & Estufa & $90,7 \mathrm{a}$ & $78,4 \mathrm{a}$ & $28,4 \mathrm{a}$ & $18,0 \mathrm{a}$ & $5,2 \mathrm{a}$ \\
\hline & Microondas $900 \mathrm{~W}$ & 90,9 a & $80,2 \mathrm{a}$ & $27,2 \mathrm{a}$ & $19,0 \mathrm{a}$ & $6,1 \mathrm{a}$ \\
\hline & Microondas $900-400 \mathrm{~W}$ & 90,3 a & $78,0 \mathrm{a}$ & $25,7 \mathrm{a}$ & $20,9 \mathrm{a}$ & 6,3 a \\
\hline \multirow{3}{*}{ T.r. } & Estufa & $90,1 \mathrm{a}$ & $76,7 \mathrm{a}$ & $19,3 \mathrm{a}$ & $21,6 \mathrm{a}$ & $5,6 \mathrm{a}$ \\
\hline & Microondas $900 \mathrm{~W}$ & $90,4 \mathrm{a}$ & $74,4 \mathrm{a}$ & $20,3 \mathrm{a}$ & $22,2 \mathrm{a}$ & $8,0 \mathrm{a}$ \\
\hline & Microondas $900-400 \mathrm{~W}$ & $90,2 \mathrm{a}$ & $76,6 \mathrm{a}$ & $21,0 \mathrm{a}$ & $19,7 \mathrm{~b}$ & $7,6 \mathrm{a}$ \\
\hline \multirow{3}{*}{ T.p. } & Estufa & $89,3 \mathrm{a}$ & $73,9 \mathrm{a}$ & $24,1 \mathrm{a}$ & $17,6 \mathrm{a}$ & $10,4 \mathrm{a}$ \\
\hline & Microondas $900 \mathrm{~W}$ & $88,8 \mathrm{a}$ & $70,3 \mathrm{a}$ & $22,1 \mathrm{a}$ & $16,2 \mathrm{a}$ & $8,2 \mathrm{a}$ \\
\hline & Microondas $900-400 \mathrm{~W}$ & 88,9 a & $74,4 \mathrm{a}$ & $22,0 \mathrm{a}$ & $17,7 \mathrm{a}$ & 9,0 a \\
\hline \multirow{3}{*}{ Th.p. } & Estufa & $89,6 \mathrm{a}$ & $67,8 \mathrm{a}$ & $12,0 \mathrm{a}$ & $37,9 \mathrm{a}$ & $6,9 \mathrm{a}$ \\
\hline & Microondas $900 \mathrm{~W}$ & $89,4 \mathrm{a}$ & $69,7 \mathrm{a}$ & $12,4 \mathrm{a}$ & $37,7 \mathrm{a}$ & $6,7 \mathrm{a}$ \\
\hline & Microondas $900-400 \mathrm{~W}$ & $89,5 \mathrm{a}$ & $63,1 \mathrm{~b}$ & $10,9 \mathrm{~b}$ & $38,5 \mathrm{a}$ & $8,7 \mathrm{a}$ \\
\hline \multirow{3}{*}{$\begin{array}{l}\text { M.s.- } \\
\text { D.glom. }\end{array}$} & Estufa & 88,3 a & 68,6 a & $25,5 \mathrm{a}$ & $28,0 \mathrm{a}$ & $4,5 \mathrm{a}$ \\
\hline & Microondas $900 \mathrm{~W}$ & 88,0 a & $67,9 \mathrm{a}$ & $24,5 \mathrm{a}$ & $26,8 \mathrm{a}$ & $6,2 \mathrm{a}$ \\
\hline & Microondas $900-400 \mathrm{~W}$ & 87,9 a & $66,5 \mathrm{a}$ & $25,2 \mathrm{a}$ & $27,1 \mathrm{a}$ & $6,2 \mathrm{a}$ \\
\hline \multirow{3}{*}{$\begin{array}{l}\text { F.arun.-T.r.- } \\
\text { D.glom. }\end{array}$} & Estufa & $86,6 \mathrm{a}$ & $74,5 \mathrm{a}$ & $12,8 \mathrm{a}$ & $35,2 \mathrm{a}$ & $6,6 \mathrm{a}$ \\
\hline & Microondas $900 \mathrm{~W}$ & 86,2 a & $75,8 \mathrm{a}$ & $12,1 \mathrm{a}$ & $35,0 \mathrm{a}$ & $7,4 \mathrm{a}$ \\
\hline & Microondas $900-400 \mathrm{~W}$ & 86,2 a & 73,9 a & $12,2 \mathrm{a}$ & $35,3 \mathrm{a}$ & $7,6 \mathrm{a}$ \\
\hline \multirow{3}{*}{ L.p.-T.r. } & Estufa & $88,5 \mathrm{a}$ & $75,0 \mathrm{a}$ & $19,5 \mathrm{a}$ & $28,4 \mathrm{a}$ & $3,7 \mathrm{a}$ \\
\hline & Microondas $900 \mathrm{~W}$ & $88,4 \mathrm{ab}$ & $75,7 \mathrm{a}$ & $16,8 \mathrm{~b}$ & $28,6 \mathrm{a}$ & $7,5 \mathrm{a}$ \\
\hline & Microondas $900-400 \mathrm{~W}$ & $88,0 \mathrm{~b}$ & $75,2 \mathrm{a}$ & $17,7 \mathrm{~b}$ & $28,6 \mathrm{a}$ & $6,7 \mathrm{a}$ \\
\hline
\end{tabular}

Para cada especie o pastura, los valores en columnas seguidos de una misma letra no difieren entre sí según Test de Duncan (p $\leq 0,05$ ). MO: materia orgánica, DIVMO: digestibilidad in vitro de la MO, PB: proteína bruta, FDA: fibra detergente ácida, NIDA: nitrógeno insoluble en detergente ácido.

M.s.: Medicago sativa; T.r.: Trifolium repens; T.p.: Trifolium pratense; Th.p: Thinopyrum ponticum; D.glom.: Dactylis glomerata; F.arun.: Festuca arundinacea; L.p.: Lolium perenne.

9; y 10 min de exposición a $900 \mathrm{~W}$ de potencia. Excepto a 10 min de exposición, en la cual el forraje se carbonizaba, en el resto no observaron diferencias de PB y FDA entre métodos de secado. Un parámetro similar como lo es la fibra detergente neutra, Karn (1991) no obtuvo diferencias en muestras de especies nativas (Pascopyrum smithii (Rydb.) Löve, Bouteloua gracilis pratensis L., Carex species, Stipa comata Trin. y Rupr., Stipa viridula Trin., Amorpha canescens Pursch, Artemisia campestris L. y Artemisia ludoviciana Nutt) secadas con estufa y microondas.

Respecto a la DIVMO, sólo se encontraron diferencias significativas $(\mathrm{p} \leq 0,05)$ en $T$. ponticum, donde $\mathrm{T} 1$ y $\mathrm{T} 2$ registraron valores mayores que T3. Por otro lado, los valores de MO no expresaron diferencias significativas $(\mathrm{p} \leq 0,05)$ entre tratamientos para todos los materiales forrajeros utilizados (Cuadro 6). Higgins y Spooner (1986) no observaron diferencias de digestibilidad in vitro de la MS en muestras de $M$. sativa secadas en horno microondas y estufa. Asimismo, Karn (1991), utilizando una estufa de circulación forzada de aire a $50^{\circ} \mathrm{C}$, no obtuvo diferencias significativas durante dos años seguidos, en el valor de DIVMO en muestras de pasturas de especies nativas secadas en la estufa respecto a las secadas en el horno de microondas.

Para los distintos materiales forrajeros utilizados no se encontraron diferencias $(p \geq 0,05)$ en los valores de NIDA bajo los tratamientos empleados (Cuadro 6). Según Goering et al. (1973), un incremento significativo en el NIDA puede ocurrir cuando las temperaturas de secado del forraje exceden $\operatorname{los} 60^{\circ} \mathrm{C}$ por un tiempo mayor a las $24 \mathrm{~h}$. Esto es resultado de la condensación de los grupos carboxilo con aminos de las proteínas, aminoácidos y otros componentes. A juzgar por los resultados aquí pre- 
sentados, en los cuales los valores de NIDA no difirieron entre tratamientos, el efecto antedicho no se habría manifestado en $\mathrm{T} 1$ si bien se realizó a $65^{\circ} \mathrm{C}$ por $48 \mathrm{~h}$.

Koenig et al. (2000) y Karn (1991) destacan las ventajas del secado rápido con el horno microondas y las posibilidades de obtener la materia seca de una muestra de forraje, sin afectar aparentemente su contenido de nitrógeno. En el mismo sentido, Marur y Sodek (1995), destacaron el bajo costo y la rapidez de uso del horno microondas, aunque no parecerían adecuadas las muestras secadas con horno microondas, para ser utilizadas en determinación de proteínas solubles. Sin embargo, destacan la utilidad que podría dar para determinar otros parámetros bioquímicos de especies vegetales.

\section{CONCLUSIONES}

Los resultados obtenidos en este trabajo permiten concluir que:

1. La calibración del método de secado con horno microondas para ser utilizados con estos materiales forrajeros implica someter el forraje a un período inicial de secado de 4 ó 5 min y fases posterio- res no mayores a 1 min de duración, de manera tal de lograr mayor exactitud y evitar el carbonizado del forraje.

2. El método de secado con horno microondas permite determinar confiablemente el contenido de MS en los materiales forrajeros utilizados, reduciendo significativamente el tiempo de secado ( 6 a $8 \mathrm{~min}$ ) respecto a la estufa de circulación forzada de aire (48 h).

3. El uso del horno microondas a $900 \mathrm{~W}$ no modificó sustancialmente los parámetros de calidad del forrage, aunque en T. repens-L. perenne la PB difirió del tratamiento testigo.

4. A 900 y $400 \mathrm{~W}$ de potencia el horno microondas tampoco produjo alteraciones importantes de calidad del forraje. Sin embargo, al utilizar materiales como T. repens, Th. ponticum o la mezcla $T$. repens-L. perenne se debería tener mayor precaución, ya que se observaron algunas diferencias entre los tratamientos.

\section{RECONOCIMIENTO}

A la Ingeniera Agrónoma Ana Beatriz Wingeyer por su constante apoyo.

\section{LITERATURA CITADA}

Agnusdei, M.G., M.R. Colabelli, y R.C. Fernández Grecco. 2001. Crecimiento estacional de forraje de pasturas y pastizales naturales para el Sudeste Bonaerense. 37 p. Boletín Técnico $\mathrm{N}^{\circ} 152$. Secretaría de Agricultura, Pesca y Alimentación, Instituto Nacional de Tecnología Agropecuaria (INTA), EEA Balcarce, Balcarce, Argentina.

ASAE. 2004. Moisture measurement - forages. S358.2. p. 584. In ASAE Standards 2004. 51 $1^{\text {st }}$ ed. American Society of Agricultural Engineers (ASAE), St. Joseph, Michigan, USA.

Benvenutti, L., e R. Cavalli. 1994. Metodologia per la determinazione rapida dell'humidita del foraggio in un centro consortile di essicazione. Riv. di Ing. Agr. (Italia) 25(1):50-55.

Bridke, D.D. 1991. Developmental morphology and physiology of grasses. p. 85-108. In Heitschmidt, R.K. and J.W. Stuth (eds). Grazing management. An ecological perspective. Timber Press, Portland, Oregon, USA.
Bruno, O.A., H. Castro, E.A. Comerón, M.C. Díaz, S. Guaita, M.C. Gaggiotti, y L.A. Romero. 1995. Técnicas de muestreo y parámetros de calidad de los recursos forrajeros. 14 p. Publicación Técnica $\mathrm{N}^{\mathrm{o}} 56$. Instituto Nacional de Tecnología Agropecuaria (INTA), EEA Rafaela, Rafaela, Argentina.

Crespo, R.J. 2002. Uso del horno microondas para la obtención del valor de materia seca en especies forrajeras. 48 p. Tesis Ingeniero Agrónomo. Universidad Nacional de Mar del Plata, Facultad de Ciencias Agrarias, Balcarce, Argentina.

Farmer, G.S., and G.H. Brusewitz. 1980. Use of home microwave oven for rapid determination of moisture in wet alfalfa. Trans. ASAE 23:170-172.

Goering, H.K., P.J. van Soest, and R.W. Hemken. 1973. Relative susceptibility of forages to heat damage as affected by moisture, temperature, and $\mathrm{pH}$. J. Dairy Sci. 56:137-143. 
Hernández Pastorini, L., M.A. Bacarin, e C. Miranda Abreu. 2002. Secagem de material vegetal em forno de microondas para determinação de material seca e analyses químicas. Ciênc. Agrotec. (Brasil) 26:12521258. Disponible en http://www.editora.ufla.br/ revista/26_6/art18.pdf Leído el 13-7-2004.

Higgins, T.R., and A.E. Spooner. 1986. Microwave drying of alfalfa compared to field-and oven-drying: Effects on forage quality. Anim. Feed Sci. Technol. 16:1-6.

Horneck, D.A., and R.O. Miller. 1998. Determination of total nitrogen in plant tissue. p. 75-83. In Kalra, Y.P. (ed.). Handbook of references methods for plant analysis. Soil and Plant Analysis Council. CRC Press, Boca Raton, Florida, USA.

Karn, J. F. 1991. Chemical composition of forage and feces as affected by microwave oven drying. J. Range Manage. 44:512-515.

Koenig, R. T., M. Winger, and B. Kitchen. 2000. Simple, low-cost data collection methods for agricultural field studies. J. Extension 38(2). Available in www.joe.org Accessed March 24, 2007.

Komarek, A.R., J.B. Robertson, and P.J van Soest. 1994. Comparison the filter bag technique to conventional filtration in the van Soest NDF analysis of 21 feeds. National Conference on Forage Quality, Evaluation and Utilization - Poster Session Abstracts. 13-15 Abril. Lincoln, Nebraska. USA.
Marur, C. J. and L. Sodek. 1995. Microwave drying of plant material for biochemical analysis. Rev. Bras. Fisiol. Veg. 7:111-114.

Narasimhalu, P., H.T. Kunelius, and K.A. Winter. 1982. Rapid determination of dry matter in grass silage of Lolium sp. using a microwave oven. Can. J. Plant Sci. 62:233-235.

Petruzzi, H.J., N.P. Stritzler, C.M. Ferri, J.H. Pagella, y C.M. Rabotnikof. 2005. Determinación de materia seca por métodos indirectos: utilización del horno a microondas. p. 8-11. Boletín de Divulgación Técnica $\mathrm{N}^{\circ}$ 88. INTA - Facultad Agronomía, Universidad Nacional de La Pampa (UNLPam), Anguil, Argentina. Disponible en http://www.inta.gov.ar/anguil/ Leído el 12 de noviembre de 2005.

Schuman, G.E., and F. Rauzi. 1981. Microwave drying of rangeland forage samples. J. Range Manage. $34: 426-428$.

Tilley, J.M., and R.A. Terry. 1963. A two stage technique for the in vitro digestion of forage crops. J. Br. Grassl. Soc. 18:104-111. 